\title{
Caminos posibles para las transformaciones educativas
}

Soc. Renato Opertti*

\begin{abstract}
El objetivo del artículo es presentar y discutir algunos de los puntos claves que se entienden como condiciones necesarias para lograr efectivas transformaciones institucionales y pedagógicas en los procesos de cambio educativo que están ocurriendo en la Educación Media Superior. En primer lugar, se hace hincapié en la necesidad de contar con marcos conceptuales unitarios (por ejemplo, los conceptos de competencias, trayectos y espacios curriculares), comunes al sistema educativo en su conjunto, a partir de los cuales se puedan diseñar estrategias exitosas de modificación curricular con capacidad de articulación hacia adentro y fuera de la Educación Media. En segundo lugar, la centralidad del centro educativo como eje sustento de la innovación se gesta a partir de una dinámica relación entre normas y criterios universales que den credibilidad, orden y transparencia al sistema, y la posibilidad real de ejercer la libertad y asumir la responsabilidad efectiva por lo actuado, desarrollando su propio traje a medida (por ejemplo, los espacios de descentralización curricular). En tercer lugar, la debida jerarquización de las respuestas sustantivamente pedagógicas, generadas a partir de las acciones de centro y áulicas, que permitan superar con respuestas de calidad y acotadas a dimensiones operables, las explicaciones burdas de determinismo contextual. En cuarto lugar, la búsqueda de la triple inclusión de los jóvenes como un actor principal del sistema educativo: en lo que tiene que ver con la comprensión antropológica de sus vivencias y percepciones, la prevención social de situaciones de pobreza y de marginalidad y la preparación para el ejercicio de una ciudadanía competente.
\end{abstract}

El objetivo de este trabajo es contribuir a reflexionar sobre objetivos y actividades en una perspectiva de largo aliento y en el marco de la búsqueda de caminos acordados de construcción colectiva. Nos planteamos una pregunta general ${ }^{1}$ : ¿Cómo lograr responder exitosamente al precepto de que el destino de las reformas o de las transformaciones, como se les guste denominarlas, se juega finalmente en los centros y en las aulas?. ¿Cuáles son los senderos que nos llevan a que verdaderamente tengamos un impacto real donde debemos tenerlo? Una mejor calidad de gestión de centro involucra lo institucional y lo pedagógico en una situación de diálogo permanente.

Vamos a referirnos a caminos y a realidades que se están construyendo como proceso, lo cual es y va a ser largo, complejo y sin resultados inmediatos en el tiempo. Intentaremos abordar los temas de la gestión a partir de la Transformación de la Educación Media Superior (TEMS) que se inició en el 2003 en once liceos (dos en Montevideo y nueve en el Interior) y seis escuelas técnicas (todas en el Interior) pertenecientes al sector público. 


\section{Marcos conceptuales unitarios}

El primer punto de reflexión a partir de nuestra pregunta general, tiene que ver con la significación y relevancia de un sólido marco conceptual que sustente la propuesta educativa. Creemos ciertamente que los problemas de gestión tienen su génesis estructural en la inexistencia de marcos conceptuales fuertes de referencia. La necesidad de pensar sobre la base de conceptos orientadores, claros en sus sentidos y significados, transversales al sistema, nos permite superar la tentación de ver el marco conceptual como reducido a un área o subsistema educativo. Una de las enormes ventajas de tener conceptos fuertes es que aumenta la capacidad de realizarlos y de ser finalmente eficaces. Una buena discusión nutre la comprensión y el sentido que finalmente se le da a los conceptos claves en las reformas y transformaciones, como categorías universales de pensamiento y de acción.

La debilidad, la atomización y el aislacionismo conceptual son un viejo problema estructural del sistema educativo formal ${ }^{2}$, en cuyo desarrollo incide la existencia de un marco organizacional fragmentado que dificulta la construcción colectiva y acordada. En la Administración Nacional de Educación Pública (ANEP) sufrimos una fragmentación que solemos expresar, de forma hasta si se quiere mágica, rutinaria y resignada, bajo el rótulo "falta de articulación". La ausencia de orientación conceptual es uno de los nudos críticos más importantes ya que compromete el logro de una visión unitaria de nuestro sistema educativo.

El armado de una matriz conceptual común al conjunto de las ofertas educativas es un camino necesariamente a transitar. En el marco de la Transformación de la Educación Media Superior (TEMS), se está haciendo el esfuerzo de pensar la estructura curricular a partir de un marco conceptual que dé significación a cada uno de los componentes de esa estructura. Partimos de una situación actual de extrema fragmentación donde por un lado, la Educación Secundaria presenta un marcado sesgo preuniversitario de finalidad básicamente propedéutica ${ }^{3}$ y por otro lado, aparece la educación técnica con variantes tecnológicas ${ }^{4}$-reformadas en 1997 , y profesionales ${ }^{5}$ muy heterogéneas.

La fragmentación conceptual tiene costos operativos fuertes que penalizan a los jóvenes. Es común que un estudiante deba empezar de nuevo en la educación técnica aun cuando haya cursado estudios en Educación Secundaria. Solemos señalar que se trata de trabas burocráticas en torno a las reválidas, pero en realidad, el fondo del problema es la falta de conceptos vertebradores que permitan articular una visión de conjunto en la Educación Media.

Creemos que el proceso de Transformación de la Educación Media Superior avanza hacia la unicidad y coherencia conceptual en la medida que plantea un diseño estructurado en torno a la idea de competencias, entendidas como el desarrollo de capacidades complejas que permitan reflexionar y actuar sobre múltiples realidades a partir de diferentes campos del saber.

Las competencias son definidas a nivel transversal del conjunto de la Educación Media Superior, incluyendo diversas ofertas. Complementariamente, se conceptualizan tres trayectos curriculares vinculados a Comunicación y Expresión; Ciencias Naturales y Matemática; Filosofía y Ciencias Sociales, que son entendidos como los recorridos curriculares que desarrollan determinadas competencias y habilidades fundamentales a través de los contenidos de las asignaturas. Las asignaturas se piensan y se ordenan en función de los logros de las competencias asociadas a cada trayecto curricular. Esto implica un cambio conceptual importante ya que es común pensar la asignatura en sí misma sin la necesaria vinculación con las demás, donde no se vislumbra la necesidad de compartir conceptos.

\section{4 - Universidad ORT Uruguay}


La búsqueda en la concreción de una visión unitaria es una forma de incentivar la comunicación fluida entre diversas ofertas, evitando que la variable de ajuste de la atomización curricular sean los jóvenes.

La combinación más adecuada entre lo particular y lo universal parece radicar en que cada oferta educativa pueda tener su camino propio dentro de un marco universal, preferiblemente a uno propio dentro de un marco atomizado. A vía de ejemplo, la Educación Tecnológica mantiene su especificidad institucional y curricular pero a la vez, comparte con otras ofertas de Educación Media Superior, un marco conceptual común de estructura curricular ${ }^{6}$. Ciertamente la unicidad contribuye a darle al sistema educativo, mayores grados potenciales de transparencia, equidad y calidad.

El sistema educativo en su conjunto debe avanzar en el desarrollo de un marco conceptual unitario, desde la Educación Inicial a la Formación Docente. La necesidad del ajuste y del perfeccionamiento curricular sobre la base de conceptos compartidos, debería ser una actividad permanente, que nos llevara a visualizar la significación de contar con unidades técnicamente sólidas de diseño, seguimiento y evaluación curricular. Hasta el presente, el sistema educativo ha preferido en general pensar e implementar los cambios curriculares a través de comisiones que no tienen la suficiente continuidad en el tiempo. No tenemos desarrollada una concepción dinámica, abierta y plural del cambio curricular, y solemos predicar una suerte de suma cero donde se combinan y confunden discusiones ideológicas, de técnica curricular y de intereses corporativos, gremiales u otros de similar tenor. Parte del problema es la ausencia de transparencia en los debates, lo cual en empobrecimiento y conformismo intelectual.

El bloqueo de las discusiones, que llevan inexorablemente a la debilidad propositiva, se traducen en la permanencia de diseños curriculares congelados en el tiempo, que parecen ser inmutables frente a los diversos tipos de cambios que se dan en las sociedades así como frente a las realidades de la implementación. Predomina la visión del currículum prescripto, que genera muchas veces aprehensión y miedo, y se ejemplifica en la idea "tengo que cumplir con el programa". El currículum, a través de los programas, prescriben un conjunto de contenidos, que deben ejecutarse, generando una fuerte y desafortunada separación entre las fases de diseño curricular y de implementación. A nuestro criterio, el problema radica en no entender el currículum como proceso, tal cual señalan Gimeno S. y Pérez Gómez ${ }^{7}$, en el cual conviven lo prescripto y regulado, las prácticas que desarrollan profesores y alumnos, la organización del currículum en un centro educativo determinado, su evaluación y reelaboración.

La idea de instituir, en el tiempo, una visión de currícula inmóvil nos parece extremadamente simplista, ingenua, y en muchos casos, puede verse y ser soberbia. Los procesos de reforma y/o transformación se desdibujan notoriamente en la medida, en que anclados en las ideas iniciales de cambio, se separan nítidamente las fases de diseño e implementación, sin tomar en cuenta la necesidad de desarrollar instrumentos ágiles y abiertos de retroalimentación nutridos de funciones de seguimiento y evaluación. Permanentemente, se debe trabajar en el diseño curricular como proceso, incorporando los resultados de los trabajos en los centros educativos. Si muchas veces, las realidades áulicas nos indican la necesidad de ajustes programáticos, debemos actuar en tiempo real, priorizando y jerarquizando las necesidades de formación de los jóvenes. 
La concepción de currículum como proceso se funda en la idea que la institución gesta y opera a muy diferentes niveles; en el trabajo cotidiano de inspectores, directores y fundamentalmente docentes, se arma la propia dinámica de la acción curricular. No es un problema de sólo coordinar la implementación a través de acuerdos normativos, sino también de gestar una concepción unitaria de currículum que tiene diferentes niveles de desarrollo y concreción en las instituciones educativas como tales. La necesidad de orientar y apuntalar el proceso curricular es un asunto crítico.

El nuevo diseño de la Educación Media Superior se orienta hacia un desarrollo curricular abierto, donde se puedan generar condiciones reales para una participación plural, profesional y sustantiva. No se trata de hacer de la participación un ejercicio retórico, sino de identificar instrumentos que permitan entender, comprender e incorporar las visiones de múltiples actores, buscando generar una síntesis creativa y contemporizadora de los diferentes pareceres. La participación se genera desde el propio diseño curricular, para que efectivamente pueda haber una real apropiación a partir de la credibilidad, del convencimiento y de la acción en común.

La incesante y afanosa búsqueda por acordar un camino de construcción colectiva implica la recreación del diálogo en su valor sustantivo. La Comisión TEMS lo promueve intensamente a muy diferentes niveles -inspectores, directores, docentes, estudiantes y sectores de la sociedad civil-, apostando a que en su desarrollo se generen propuestas acordadas y sustentables. Los encuentros cara a cara con los jóvenes constituyen una forma de entender sus necesidades y expectativas, siendo fundamentales las capacidades de escucha y de comprensión.

La convocatoria al diálogo para renovar la currícula es fundamental ya que de lo contrario, seguimos atrapados por la existencia de divisiones tajantes entre diseñadores y ejecutores. Esta separación se proyecta en la imagen, por un lado, de tecnócratas que piensan los cambios en forma solitaria y descontextualizada y por otro lado, de docentes que tienen que aplicarlos, constatando muchas veces, que lo que se prometió y se dijo no tiene nada que ver con lo que se hace en la práctica. Si actores claves no creen en lo que hacen y no participan entusiasta y profesionalmente desde un principio, no se van a lograr prácticas exitosas para mejorar la calidad de las enseñanzas y de los aprendizajes.

Si no hay creencias sustentables en el tiempo y si efectivamente no nos apropiamos de los cambios en un sentido inclusivo y plural, de nada vale sólo realizar inversiones significativas en obras físicas y en equipamientos educativos. Falta un elemento insustituible: el convencimiento docente para generar cambios reales.

La apuesta al diálogo como proceso contribuye centralmente al desarrollo y la concreción de propuestas que se han ido acordando a través de sucesivas reformulaciones. A vía de ejemplo, la participación profesional y comprometida de cuerpos inspectivos de Educación Media, ha permitido incorporar un conjunto de propuestas que incluyen, entre otros aspectos relevantes, nuevas orientaciones y énfasis en la Educación Media General tales como el bachillerato en arte y comunicación, en lenguas extranjeras y ciencias de la vida y la salud con énfasis en deportes o en recursos naturales y medio ambiente ${ }^{8}$; asimismo, la inclusión de nuevas asignaturas como Crítica de los saberes, Ciencias de la tierra y del espacio, Geografía y Economía. 
La clave de estos aportes al igual que de otros, radica en que los mismos se sustentan en salas docentes y en instancias similares donde de manera progresiva y constructiva, se elaboraron, acordaron y perfeccionaron ideas y propuestas. Se trata esencialmente de un trabajo en colaboración que involucra múltiples actores a muy diferentes niveles. Cuando la gente siente que hay espacios para aportar, lo hace, en la mayoría de los casos, de manera apasionada y positiva, brindando generosamente sus tiempos y sus saberes al proceso de construcción colectiva. Todos tenemos la oportunidad de vencer prejuicios que suelen bloquear y postergar debates y acuerdos sustantivos.

Esperamos que la recreación del diálogo como base de la construcción colectiva, nos empuje decididamente hacia la búsqueda de estrategias que permitan mejorar las condiciones de enseñanza y de aprendizaje, tomando como eje estructurador las expectativas y las necesidades de los jóvenes. Todos sabemos de la existencia de corporativismos y de las formas en que los mismos influyen en la sociedad, pero es siempre posible dialogar sobre la base del respeto y de la confianza mutua así como de la predisposición a acordar. Generalmente, las ausencias en la comunicación las pagan severa e injustamente los jóvenes, hipotecando sus oportunidades de desarrollo individual y social.

El fortalecimiento del diálogo hacia el interior del sistema educativo, se ha ido acompañando de la incorporación de otras miradas sobre la transformación, provenientes principalmente de las universidades y de diferentes sectores y personalidades de la sociedad civil vinculados por ejemplo al arte, al deporte y a la música. Particularmente, la Universidad de la República parece visualizar este proceso como de larga duración, en el cual su efectiva participación tiene, por lo menos, dos niveles: por un lado, el reconocimiento de la necesidad de revisar conceptualmente un modelo de bachillerato secundario excesivamente orientado hacia una finalidad propedeútica, de atiborrado contenido temático que no ambienta el desarrollo de competencias fundamentales que son el cimiento de buenos aprendizajes en la educación universitaria; y por otro lado, su centralidad en el apuntalamiento de programas de mejoramiento de la calidad docente, poniendo particular énfasis en la actualización disciplinar.

Buena parte del diálogo constructivo con las Universidades parte de reconocer que la Educación Media como tal tiene una identidad y una especificidad propia en cuanto a su misión y a sus objetivos, y que no es bueno que actúe solamente por demandas agendadas por la Educación Terciaria.

\section{La centralidad del centro educativo}

Es innegable que cada centro tiene una misión a definir y a cumplir dentro de un marco conceptual unitario. De manera progresiva, se ha ido superando la idea del centro como una entidad autártica, cuyo desempeño mejora sustancialmente o bien por la transferencia de responsabilidades financieras y técnicas desde los niveles centrales a los locales, a través de esquemas ingenuos de descentralización; a través de la privatización de servicios por la vía del subsidio a la oferta o a través de bonos a las familias. Los instrumentos por sí solos no generan mejores resultados educativos si no son sustentados en estrategias que permitan fortalecer la capacidad de pensar y de actuar de los centros educativos. Una de las consecuencias más visibles de la ola descentralizadora o privatizadora, que impacta América Latina desde la década de los ochenta ${ }^{9}$, es el debilitamiento de la capacidad de propuesta y de control por parte del estado, así como cierta esquematización simplista de la capacidad autónoma y de potencial de desempeño de los centros educativos. 
El tema central parece radicar en mejorar las condiciones en que un centro educativo desarrolla una respuesta local a partir de un marco universal, el cual debe oficiar como una condición que ambiente y facilite respuestas innovadoras y audaces. El traje a medida de cada centro es posible si hay reglas que den transparencia y equidad al sistema. No confundamos la necesaria conformación de un marco orientador y regulador de iniciativas y actividades con la existencia de trabas y rigideces que inhabilitan la innovación y la creatividad en los niveles locales, provocando que la agenda de los niveles centrales de decisión esté dominada por autorizar el desarrollo de acciones que deberían estar en las competencias propias de cada centro educativo. En general, prima la concepción y la actuación sobre los controles formales pero no sobre los sustantivos.

Las rigideces institucionales provocan, entre otras cosas, que no se desarrolle la iniciativa propia en los ámbitos locales porque se está razonablemente preocupado por sus consecuencias negativas. La asfixia normativa que sufren los centros se acompaña de un preocupante vaciamiento conceptual acerca de cómo orientar las acciones educativas desde los niveles más centrales del sistema educativo.

La especificidad de la misión de cada centro educativo implica una elaboración y una construcción de una realidad sobre la que se busca incidir, dentro de entornos y marcos que deben ser comprendidos. Las múltiples situaciones que se viven en cada centro son un claro reflejo de la diversidad humana, en la cual estudiantes, directores, docentes y funcionarios administrativos convergen en un conjunto de principios y orientaciones a desarrollar. No debemos tenerle miedo a la diversidad, sino todo lo contrario; es, en efecto, un factor promotor del ejercicio de la libertad, así como también permite alcanzar mayores niveles de equidad. No vamos a incrementar la equidad por la vía de pensar que la igualdad de oportunidades se gesta a través de propuestas homogeneizadoras.

Lamentablemente, nuestro sistema educativo no promueve suficientemente la libertad, y como correlato, queda poco espacio para la innovación y la creatividad. El ejercicio ciudadano de derechos y responsabilidades debe ambientarse desde el momento en que la niña o el niño ingresan a la Educación Inicial, y en este sentido, hay una necesidad muy fuerte de mejorar, principalmente en la Educación Media, el relacionamiento cualitativo entre directores, docentes y estudiantes, sobres bases efectivas de confianza y de credibilidad mutua. Sin esas bases fortalecidas, difícilmente pueda haber una genuina y provechosa apropiación del conocimiento.

En el marco de la transformación de la Educación Media Superior, se trabaja sobre la idea de que el centro es el eje central del cambio. Algunos ejemplos concretos pueden ilustrar esta preocupación: uno es la introducción de un espacio curricular descentralizado donde el centro puede definir y desarrollar, por un mínimo de cuatro horas semanales, actividades vinculadas entre otros aspectos, a la producción escrita y oral, al deporte, a la recreación, a la música, al arte y a la informática.

La concepción de espacio curricular descentralizado radica en que el centro lo va definiendo y completando en función de la misión y de los objetivos que persigue, buscando el acuerdo y la participación del conjunto de actores involucrados. En gran medida, es un espacio de construcción ciudadana donde se ejerce la democracia con sus derechos y obligaciones. El fortalecimiento del diálogo en el propio centro educativo es una apuesta al logro de acuerdos colectivos de trabajo sobre la base de fines y objetivos consensuados. 
La capacidad de ampliar las oportunidades de trabajo por parte del centro educativo no sólo se circunscriben a los desarrollos que habilitan los espacios curriculares, sino también incluyen la idea, por ejemplo, de disponer de las condiciones y los tiempos necesarios como para apoyar fuertemente los aprendizajes de los alumnos, y en general, brindar y compartir elementos de orientación personal y profesional con los jóvenes, ya sea a través del trabajo de los adscriptos o de los docentes de aula. O en otro plano, la propuesta de diseñar e implementar proyectos comunitarios donde existan posibilidades reales para ejercer la solidaridad.

El énfasis puesto en dotar a los centros de recursos ${ }^{10}$, proyectos e instrumentos para que puedan llevar a cabo su misión institucional, es en gran medida, una respuesta necesaria a las complejidades de vida de los jóvenes para quienes el nudo crítico de los aprendizajes no puede únicamente encararse a través de la relación con las asignaturas. La ausencia de encuadre orientador de las actividades de los jóvenes daña enormemente las posibilidades de lograr procesos y resultados adecuados de aprendizaje. En la encuesta realizada a estudiantes de Educación Media Superior en el 2002 (liceos y escuelas técnicas en el sector público) ${ }^{11}$, un tercio sostuvo que no recibió orientación alguna en el centro educativo en los aspectos educativos y profesionales, y asimismo, otro tercio la calificó de insuficiente.

\section{Las respuestas sustantivamente pedagógicas}

La puesta en real dimensión de los enfoques pedagógicos en la gestión de los centros educativos nace de la necesidad de entender la especificidad de la respuesta que se debería dar frente a los problemas del enseñar y el aprender. No se trata únicamente de obtener y acumular recursos e insumos de infraestructura física, de informática y de materiales didácticos, sino de también ver cómo se gestionan, desde el compromiso, la profesionalidad y la creatividad de los actores del centro educativo, para buscar obtener mejores resultados. Hay cierta presencia de un pensamiento mágico que puede operar en un doble sentido negativo: por un lado, suponer que si no hay mejores condiciones de infraestructuras y de equipamiento, no hay logros relevantes en los aprendizajes; y por otro lado, dar por cierto que si no tenemos buenos indicadores de funcionamiento (por ejemplo, un número de alumnos por grupo que supere determinados niveles considerados adecuados), tampoco se obtendrán buenos resultados. Es necesario trabajar intensamente y con programas de larga duración en el mejoramiento de las condiciones de funcionamiento de los centros educativos ${ }^{12}$, que sean precisamente un motivador central y una oportunidad real para mejorar la calidad de la educación, pero no es lo único relevante.

Es necesario también superar las explicaciones de burdo sociologismo que terminan por establecer un determinismo contextual que poco contribuye al conocimiento sustancial de los procesos y de los resultados educativos. Los estudios realizados por el Programa MESYFOD (Modernización de la Educación Secundaria y la Formación Docente) ${ }^{13}$, a partir del Censo de Aprendizajes aplicado a los 3eros. años de Ciclo Básico de Educación Media, señalan qué lejos estamos de una explicación monocausal de los desempeños por la sola presencia o ausencia de determinados niveles culturales y socioeconómicos de las familias. Sin dejar de indicar la relevancia explicativa de los factores contextuales de tipo social, se debe también ver y dimensionar la incidencia de aquellos vinculados a las redes familiares, más precisamente, a las expectativas de los padres sobre el desempeño de sus hijos, al relacionamiento cualitativo entre padres e hijos o bien a que padres e hijos compartan un proyecto educativo común hacia el futuro. 
Por otra parte, el determinismo contextual oscurece muchas veces las diferencias que se dan en el interior de zonas de perfil socioeconómico similar, lo cual alimenta la proliferación de actitudes cargadas de pesimismo y de resignación. Se sabe por ejemplo, que hay liceos públicos en Montevideo localizados en zonas tipificadas como carenciadas, que tienen tasas muy bajas de repetición y de deserción, similares a la que se registran en liceos públicos de zonas de nivel socioeconómico medio o alto. La similitud en los resultados educativos entre liceos con perfiles socio-económicos muy distintos, debería motivar a tener aun más en cuenta los factores de gestión institucional y pedagógica que pueden marcar la diferencia. En gran medida, resulta necesario considerar y respetar la evidencia empírica que resulta de ver y sistematizar las buenas prácticas de gestión entendidas en un sentido amplio, lo cual nos permite superar enfoques excesivamente entroncados en planteamientos retóricos y especulativos.

La evidencia empírica como sustento de planes de mejoramiento de calidad, supone integrar en la práctica, visiones y enfoques metodológicos, donde triangulemos perspectivas y datos a partir de técnicas cualitativas y cuantitativas. Por ejemplo, saber leer un cuadro, realizar entrevistas en profundidad a informantes claves, graficar una tendencia y observar el comportamiento de los alumnos en el aula, son habilidades complementarias que nos alfabetizan y nos hacen competentes para poder analizar e incidir en la realidad. Los prejuicios metodológicos sólo conspiran contra el logro de una mirada plural y amplia de los fenómenos educativos.

En esta línea de apertura metodológica, se está trabajando con el cuerpo inspectivo de Educación Secundaria y Técnico-Profesional, en el diseño de un sistema de indicadores de autoevaluación institucional, que se entiende como un componente crítico de la propuesta de evaluación de la Educación Media Superior. La idea central es que el centro construya un conjunto de indicadores que le van permitir autoevaluar su gestión desde perspectivas metodológicas complementarias, observando y analizando si el mismo logra cumplir los objetivos que se trazó. Sobre la base de un análisis integrado que involucre a los diferentes actores del centro, se estará en condiciones de informar a la comunidad de lo actuado y lo alcanzado. Por ejemplo, ¿En qué medida logramos los objetivos que nos planteamos?, ¿Qué aspectos sustantivos no abordamos? y ¿Cuáles fueron los aspectos críticos en la implementación de nuestro plan de acción?. No se trata de rendir cuentas con la intencionalidad de culpabilizar y penalizar, sino de generar condiciones y espacios adecuados para que podamos intercambiar pareceres y datos sobre los objetivos y las actividades del centro, con la finalidad de mejorar la calidad de los procesos y de los resultados.

Una mirada de largo aliento nos señala que la ANEP en su conjunto, debería asumir el desarrollo de un sistema de indicadores que permitan la auto-evaluación institucional de manera permanente. El logro de una gestión eficiente en la asignación y en el uso de los recursos así como socialmente eficaz en atender las necesidades de nuestros estudiantes, sólo es sustentable si los procesos de toma de decisiones se fundan en buenos sistemas de información.

\section{La afanosa búsqueda por la inclusión de nuestros jóvenes}

Nuestra Educación Media se caracteriza porque entran muchos, retenemos a pocos y egresa una ínfima minoría. A título ilustrativo, sobre la base de un estudio de la cohorte de estudiantes que ingresó a 1er. año en 1996 a liceos públicos en turnos diurnos, solamente el 20\% estaba cursando 6to. año en el $2001^{14}$. El enorme esfuerzo de democratización matricular que se constata principalmente a partir de la segunda mitad de la década de los noventa ${ }^{15}$, es inconcluso en la medida que no logramos equidad en los egresos. La agenda educativa debe estar esencialmente motivada por la inclusión de nuestros jóvenes, que es en definitiva, una de las mayores y más firmes estrategias preventivas frente a las situaciones de pobreza y marginalidad.

\section{0 - Universidad ORT Uruguay}




\section{La inclusión tiene por lo menos tres aspectos a atender:}

a) La inclusión social, es atender la significativa proporción de jóvenes que dejan de estudiar sin haber finalizado el ciclo que cursaban; ésta es más alta que la que se registra por ejemplo en Argentina y Chile. Según estudios realizados a partir de la Encuesta de Hogares del Instituto Nacional de Estadística (INE), se estima a que a nivel del país urbano, hay aproximadamente 18.000 jóvenes en las edades de 16 a 19 años de edad que no estudian ni trabajan ni buscan trabajo, de los cuales 12.000 son mujeres ${ }^{16}$. La exclusión de género tiene que ver, entre otras cosas, con la maternidad adolescente y con hogares inestables (sin presencia de padre), donde se gestan las bases críticas del ciclo reproductor biológico y social de la pobreza. ¿Acaso no debemos preguntarnos acerca de si las ofertas actuales son buenas respuestas frente a las expectativas y necesidades de este grupo de población?

b) La inclusión antropológica, es conocer los valores, las vivencias, las percepciones, las actitudes y las motivaciones que permean la vida de los jóvenes. Desde el mundo adulto, se deben buscar formas de entender a los jóvenes, ya no simplemente como una categoría estadística. El sistema educativo adolece de conocimiento cultural sobre los jóvenes. ¿Cuántas realidades de culturas juveniles hay hoy en día? ¿Cuánto sabemos de los que los jóvenes les preocupa y les motiva? Sabemos sí cuantos pierden exámenes, cuántos repiten y cuántos desertan pero en realidad de la antropología juvenil conocemos poco.

c) La inclusión económica, es proporcionar el desarrollo de las herramientas necesarias para que puedan incorporarse a un mundo cada vez más competitivo y cruel. Deberíamos entender que el valor de la inclusión de los jóvenes es también el valor de la competitividad económica de un país que se marca a la larga por la calidad de la formación de sus recursos humanos.

En particular, la inclusión antropológica tiene que ver con el desarrollo del diálogo como espacio de construcción colectiva en el interior del centro educativo. ¿Cómo logramos fomentar un ejercicio responsable y creativo de la ciudadanía? Creemos que en los Bachilleratos Secundarios, tiende a predominar un modelo frontal de relacionamiento entre docentes y estudiantes, que parece sintetizarse en la idea de que: "vamos a clase, recibimos información, anotamos en un cuaderno, concurrimos a un profesor particular, nos sacamos las dudas y preparamos el examen". Si efectivamente prima esta idea en las percepciones de los jóvenes, no se generan condiciones para dialogar y apropiarse del conocimiento y estamos asimismo, muy lejos de cimentar bases sólidas para el ejercicio y la inclusión ciudadana.

Sabido es que esa ausencia de diálogo sustantivo no es estimulado por la existencia de liceos cuya infraestructura física y condiciones de funcionamiento (por ejemplo, grupos superpoblados) distan de ser buenas. La transformación de la Educación Media Superior está concretando el desarrollo de un plan intenso en inversiones en obra física, equipamientos mobiliario, informático y de materiales didácticos, para efectivamente mejorar las condiciones actuales de funcionamiento. La respuesta central y crítica radica en que estudiantes, docentes, directores y demás funcionarios estén más horas en los centros educativos haciendo cosas muy variadas, lo cual ambienta posibilidades para fortalecer el diálogo sobre aspectos sustantivos vinculados a la afectividad y al conocimiento. Nos preguntamos si en realidad ¿podemos pensar en estrategias que nos permitan la inclusión de los jóvenes?

En definitiva, estamos hablando de una triple inclusión: social para prevenir la pobreza y la marginalidad; antropológica para dialogar con los jóvenes acerca de sus vivencias y sus percepciones, compartiendo miedos, conflictos y proyectos presentes y futuros, y económica 
para gestar y consolidar una estrategia de mejora de la competitividad.

\section{Bibliografía y notas}

ANEP-CO.DI.CEN., Comisión TEMS. 2002. Rosalía Barcos. Módulo II El cambio curricular. Materiales de consulta y profundización. Curso de actualización para docentes de la Transformación de la Educación Media Superior. Primera Fase. Montevideo: TEMS.

ANEP-CO.DI.CEN, Comisión TEMS. 2002. Cuaderno de Trabajo, Nro. 13. Informe preliminar de los resultados de la Consulta Nacional a Estudiantes de Educación Media Superior. Montevideo: MEMFOD.

ANEP-CO.DI.CEN., MEMFOD. 2002. Aportes al seguimiento del Plan 1996 del Ciclo Básico de Educación Media: un estudio de cohortes de estudiantes. Cuadernos de Trabajo, Nro. V. Serie de Estudios Sociales sobre la Educación. Montevideo: MEMFOD.

ANEP-CO.DI.CEN., MEMFOD. 2002. Un análisis acerca de los jóvenes que no trabajan ni estudian. Cuaderno de Trabajo, Nro. VIII. Serie de Estudios Sociales sobre la Educación. Montevideo. MEMFOD.

ANEP-CO.DI.CEN., MEMFOD. 2003. Censo Nacional de Aprendizajes de los Terceros Años del Ciclo Básico de Educación Media, 1999. Resultados y desafíos. Novena comunicación. Montevideo: MEMFOD.

ANEP-CO.DI.CEN.,MEMFOD. 2003. Informe Anual de Actividades y Resultados 2003. Montevideo: MEMFOD.

BID. 1996. ¿Qué rumbo debe tomar el mejoramiento de la Educación en América Latina? En Seminario sobre Reforma Educativa. Buenos Aires: BID.

Gimeno, S., Pérez Gomez. 1993. Comprender y transformar la enseñanza. Madrid: Ediciones Morata.

OPP, BID. 1992. Diagnóstico sectorial en Educación. Tomo I. Montevideo: CT/OPP/BID.

${ }^{1}$ Fruto de las conversaciones con la Mag. María Inés Vázquez, Coordinadora Académica de Planificación y Gestión Educativa, Instituto de Educación, Universidad ORT.

${ }^{2}$ Este tema ya fue abordado a principios de la década de los noventa en un diagnóstico realizado por la Oficina de Planeamiento y Presupuesto (OPP) a través de un fondo de cooperación técnica no reembolsable (donación) proveniente del Banco Interamericano de Desarrollo (BID). Véase OPP, BID. 1992. Diagnóstico sectorial en Educación. Tomo I. Montevideo: CT/OPP/BID.

${ }^{3}$ El Plan vigente de Bachilleratos Secundarios data de 1976 y en realidad reproduce el marco conceptual del Plan 1941, el cual estaba básicamente orientado a la preparación de sectores de clase media y alta para que pudieran cursar estudios universitarios. Es bien distinto pensar las finalidades sociales y educativas del ciclo medio superior cuando se democratiza 
crecientemente el ingreso al mismo.

${ }^{4}$ A partir del año 1997, se pusieron en marcha los Bachilleratos Tecnológicos con el doble objetivo de preparar personal de nivel medio para desempeñarse en áreas entendidas como claves en el desarrollo del país, y asimismo, habilitar la realización de estudios terciarios. Al año 2003, los Bachilleratos Tecnológicos cubren 12.544 alumnos.

${ }^{5}$ Nos estamos refiriendo a la Formación Profesional Superior (FPS).

${ }^{6}$ Eso implica, entre otros aspectos, un ordenamiento de la oferta en función de competencias comunes a adquirir por trayecto curricular, así como la ubicación de las asignaturas dentro de espacios curriculares compartidos. Estos últimos son los de razonable equivalencia (objetivos comunes de formación para Educación Secundaria y Técnica), exploratorio, específico (propio de cada orientación o especialidad) y descentralizado.

${ }^{7}$ Véase Gimeno S., Pérez Gomez. 1993. Comprender y transformar la enseñanza. Morata: Madrid. Citado en ANEP-CO.DI.CEN., Comisión TEMS. 2002. Rosalía Barcos. Módulo II "EI cambio curricular". Materiales de consulta y profundización. Curso de actualización para docentes de la Transformación de la Educación Media Superior. Primera Fase. Montevideo: TEMS.

${ }^{8}$ Los énfasis, que se desarrollan a partir del 3er. año en la Educación Media General, se entienden como formas de captar los intereses de los jóvenes cuya finalidad es atraer al estudiantado con contenidos diversos que permitan la retención de la mayoría de ellos.

${ }^{9}$ Véase, por ejemplo, BID. 1996. ¿Qué rumbo debe tomar el mejoramiento de la Educación en América Latina?. En Seminario sobre Reforma Educativa. Buenos Aires: BID.

${ }^{10}$ Por ejemplo, los ERMAs (Espacios de Recursos Múltiples para el Aprendizaje) son entendidos como ámbitos que propician el acceso a diversas fuentes del conocimiento a través del uso de libros y materiales didácticos, recursos audiovisuales y computadoras con acceso a INTERNET. La idea es que docentes y estudiantes, con los asesoramientos pedagógicos debidos, usen este espacio como parte sustantiva de los procesos de enseñanza y de aprendizaje.

11 Véase ANEP-CO.DI.CEN.,TEMS. 2002. Cuaderno de Trabajo, Nro. 13. Informe preliminar de los resultados de la Consulta Nacional a Estudiantes de Educación Media Superior. Montevideo: MEMFOD.

${ }^{12}$ Entre los años 1996-2003, los Programas de Educación Media cofinanciados por el BID, han licitado por más de U\$S 100.000.000 con destino a obra física, equipamientos mobiliarios, informáticos y educativos y textos. Véase ANEP-CO.DI.CEN., MEMFOD. 2003. Informe Anual de Actividades y Resultados, 2003. Montevideo: MEMFOD.

${ }^{13}$ Nos estamos refiriendo a un total de 16 estudios que se pueden consultar en la Página Web de MEMFOD, www.memfod.edu.uy. El compendio de estudios pueden consultarse en ANEPCO.DI.CEN., MEMFOD. 2003. Censo Nacional de Aprendizajes de los Terceros Años del Ciclo Básico de Educación Media, 1999. Resultados y desafíos. Novena comunicación. Montevideo: MEMFOD.

14 Véase ANEP-CO.DI.CEN., MEMFOD. 2002. Aportes al seguimiento del Plan 1996 del Ciclo Básico de Educación Media: un estudio de cohortes de estudiantes. Cuadernos de Trabajo, 
Nro. V. Serie de Estudios Sociales sobre la Educación. Montevideo: MEMFOD.

${ }^{15}$ Entre los años 1995 y 2002 la matrícula pública del Ciclo Básico en su conjunto aumentó en cerca de 32.000 alumnos, de 110.521 en 1995 a 142.498 en el 2002.

${ }^{16}$ Véase ANEP-CO.DI.CEN., MEMFOD. 2002. Un análisis acerca de los jóvenes que no trabajan ni estudian. Cuaderno de Trabajo, Nro. VIII. Serie de Estudios Sociales sobre la Educación. Montevideo: MEMFOD.

* Master en Investigación Educativa, CIEP-IDRC, Canadá. Sociólogo. Consultor en Políticas Sociales y Educación. Coordinador, Programas de Educación Media y Formación Docente, ANEP. Docente e investigador, Instituto de Educación. 